\title{
Effect of Leptospirosis on the Concentrations of Streptomycin in Liver and Kidneys of Pigs
}

\author{
${ }^{1}$ Labrini V. Athanasiou, ${ }^{1}$ Vassilios Papatsiros, ${ }^{2}$ George Valiakos, \\ ${ }^{3}$ Dimitra Pardali, ${ }^{2}$ Antonia Touloudi, ${ }^{4}$ Vassiliki Spyrou and ${ }^{2}$ Charalambos Billinis \\ ${ }^{1}$ Department of Medicine, Faculty of Veterinary Medicine, University of Thessaly, Karditsa, Greece \\ ${ }^{2}$ Department of Microbiology and Parasitology, Faculty of Veterinary Medicine, University of Thessaly, Karditsa, Greece \\ ${ }^{3}$ Diagnostic Laboratory, Faculty of Veterinary Medicine, Aristotle University of Thessaloniki, Thessaloniki, Greece \\ ${ }^{4}$ Department of Animal Production, Technological and Educational Institute of Larissa, Greece
}

Received 2013-04-21, Revised 2013-05-14; Accepted 2013-05-28

\begin{abstract}
Leptospirosis is a re-emerging infectious disease with zoonotic potential. Pigs are reservoir hosts of the pathogen and streptomycin is among the antibiotics proposed to eliminate leptospiral infection. The purpose of this study was to determine streptomycin concentrations and the possible effect of the presence of Leptospira in the liver and kidneys on drug distribution. Six pigs with leptospirosis and six age, gender and breed matched healthy pigs were intramuscularly administered streptomycin at a dose of $10 \mathrm{mg} \mathrm{kg} \mathrm{kg}^{-1}$ bodyweight once daily for four consecutive days. Prior to administration biochemistry was employed to evaluate liver and kidney function. All pigs were euthanized and the concentrations of streptomycin in their liver and kidney were determined employing an HPLC-MC method. Infected pigs showed hyperbilirubinaemia and an increase in alanine aminotransferase and alkaline phosphatase activities compared with uninfected controls. Urine specific gravity was reduced in infected pigs, however, without elevated levels of urea and creatinine. A higher mean concentration in both organsstatistically significant only for liver-of healthy pigs was observed. The decreased concentrations of streptomycin in infected pigs could be at least partially attributed to the hepatic and renal damage caused by leptospirae and in turn the possible reduced efficacy of streptomycin to its reduced concentrations at the main sites of infection. Possible alterations of drug concentrations by liver and/or kidney infection by various pathogens including Leptospira should be taken into consideration when determining doses to result in effective drug concentrations at the site of infection.
\end{abstract}

Keywords: Leptospira, Streptomycin, Kidney, Liver, Pig

\section{INTRODUCTION} emerging infectious disease among animals and humans with an increasing trend of prevalence. However, humans are rarely chronic carriers acquiring the infection through occupational, recreational or avocational exposures. To the contrary, cattle and pigs are reservoir hosts of leptospirosis due to the chronic infection of their renal tubules and considered to be maintenance hosts or
Leptospirosis has recently been recognized as a re-

chronic carriers; pigs may harbor L. pomona, $L$. tarassovi, L. bratislava and L. muenchen, the last two being closely related (Bolin and Cassells, 1992; Burriel et al., 2003; Levett, 2001).

Virulent organisms in a susceptible host multiply in the small blood vessel endothelium, gain rapid access to the bloodstream through the lymphatics, resulting in leptospiremia and spread to all organs including kidneys, liver, lungs and skin. Leptospirosis is uncommon in suckling piglets, while a chronic low-grade disease is Corresponding Author: Labrini V. Athanasiou, Department of Medicine, Faculty of Veterinary Medicine, University of Thessaly, Karditsa, Greece 
more common in sows characterized by abortions, stillbirths and increased number of weak and non-viable piglets (Bolin and Cassells, 1990). Paired acute and convalescent serum specimens as well as PCRs are used to confirm the diagnosis (Naigowit et al., 2000).

Regarding treatment of leptospirosis in pigs, streptomycin is among the antibiotics proposed to eliminate leptospiral infection (Alt and Bolin, 1996; Dobson, 1974). The systemic administration of streptomycin is considered to be of great value in acute leptospiral infections in pigs. Streptomycin is indicated for the treatment of leptospirosis in pigs and cattle, entering the bacterial cell and combining irreversibly with ribosomal RNA (Chakraborty et al., 2011). Liver and kidney, the major organs of drug metabolism, are invaded by Leptospira spp., thus, drug disposition may be altered, resulting in suboptimal concentrations in these two organs.

The purpose of this study was to gain insight into the concentrations streptomycin against Leptospira spp. species at the liver and kidneys both in healthy pigs and in pigs with leptospirosis and to evaluate the adequacy of this concentration at the site of infection.

\section{MATERIALS AND METHODS}

\subsection{Legislative Requirements}

Experimentation on animals was in accordance with the European Communities Council Directive 86/609/EEC and National Legislation (1197/81 and 2015/92) and after receiving license for the experimentation by the State Competent Authorities.

\subsection{Serology Testing}

Serologic screening was performed in paired blood samples of pigs from suspected for leptospirosis farms with an indirect immunofluorescence assay (FULLER, USA) applying fluorescein isothiocynate anti-pig IgG conjugate (VMRD, USA) according to the manufacturer with slight modifications. Samples were considered as positive when a fourfold increase in antibody concentration occurred between the two samplings.

\subsection{Study Design}

Urine samples were selected from a farm with seropositive to Leptospira pigs to detect Leptospira by PCR. Six PCR positive for Leptospira pigs and six age, gender and breed matched healthy pigs were used in the study. To evaluate liver and kidney function, biochemistry, [urea nitrogen (BUN), creatinine, total bilirubin, alkaline phosphatase, alanine aminotransferase] and urine specific gravity were determined in pig blood and urine samples, respectively. Streptomycin was intramuscularly administered at a dose of $10 \mathrm{mg} \mathrm{kg}^{-1}$ b.w. once daily for four consecutive days. All pigs were euthanized $24 \mathrm{~h}$ after last administration and their livers and kidneys were collected. Each organ was separated in two aliquots; one was used for the detection of Leptospira spp and the other for the determination of the concentration of streptomycin.

\subsection{PCR Detection of Leptospira SPP}

DNA was isolated from $10 \mathrm{mg}$ of each tissue and urine sample using a commercial extraction DNA isolation cell and tissue kit (Puregene, Gentra Systems, USA). The sequence of the primers and the PCR conditions to amplify a part of 285 bp fragment with primers G1/G2 or a $563 \mathrm{bp}$ fragment with primers B64-I/B64-II of DNA from all pathogenic Leptospira spp., were the same as previously described (Gravekamp et al., 1993). As negative control, DEPC-treated $\mathrm{H} 2 \mathrm{O}$ was used instead of DNA in PCR assay, to exclude any contamination.

\subsection{Determination of Streptomycin Concentration in Tissues}

The concentrations of streptomycin in liver and kidney from each animal were determined employing a previously validated reverse-phase HPLC method coupled with mass spectrometry. Each tissue sample was homogenized with phosphate buffer and hexanosulfonic acid solution in a polypropylene tube. After shaking and centrifugation, the supernatant was transferred to a clear tube and the process was repeated. Finally the two extracts were transferred to a Solid Phase Extraction (SPE) manifold for the cleanup step. The eluent was evaporated under a stream of nitrogen. The residue was re-dissolved in the final solvent, filtered and injected in the LC/MS system. Column flow was diverted to waste, in the beginning and at the end of each analytical run. The validated limits of detection for kidney and liver were $249.8 \mu \mathrm{g} \mathrm{kg}^{-1}$ and $132.7 \mu \mathrm{g} \mathrm{kg}^{-1}$ respectively.

\subsection{Statistical Analysis}

The concentrations of streptomycin in liver and kidneys were compared between the two groups using Independent Samples Mann Whitney U Test. The level of significance was set at 0.05 and all tests were performed using SPSS 20.0 (SPSS Inc., Chicago, IL, USA).

\section{RESULTS AND DISCUSSION}

Two of the infected pigs were PCR positive in liver and kidneys after treatment, while two more were only PCR positive in kidneys. 

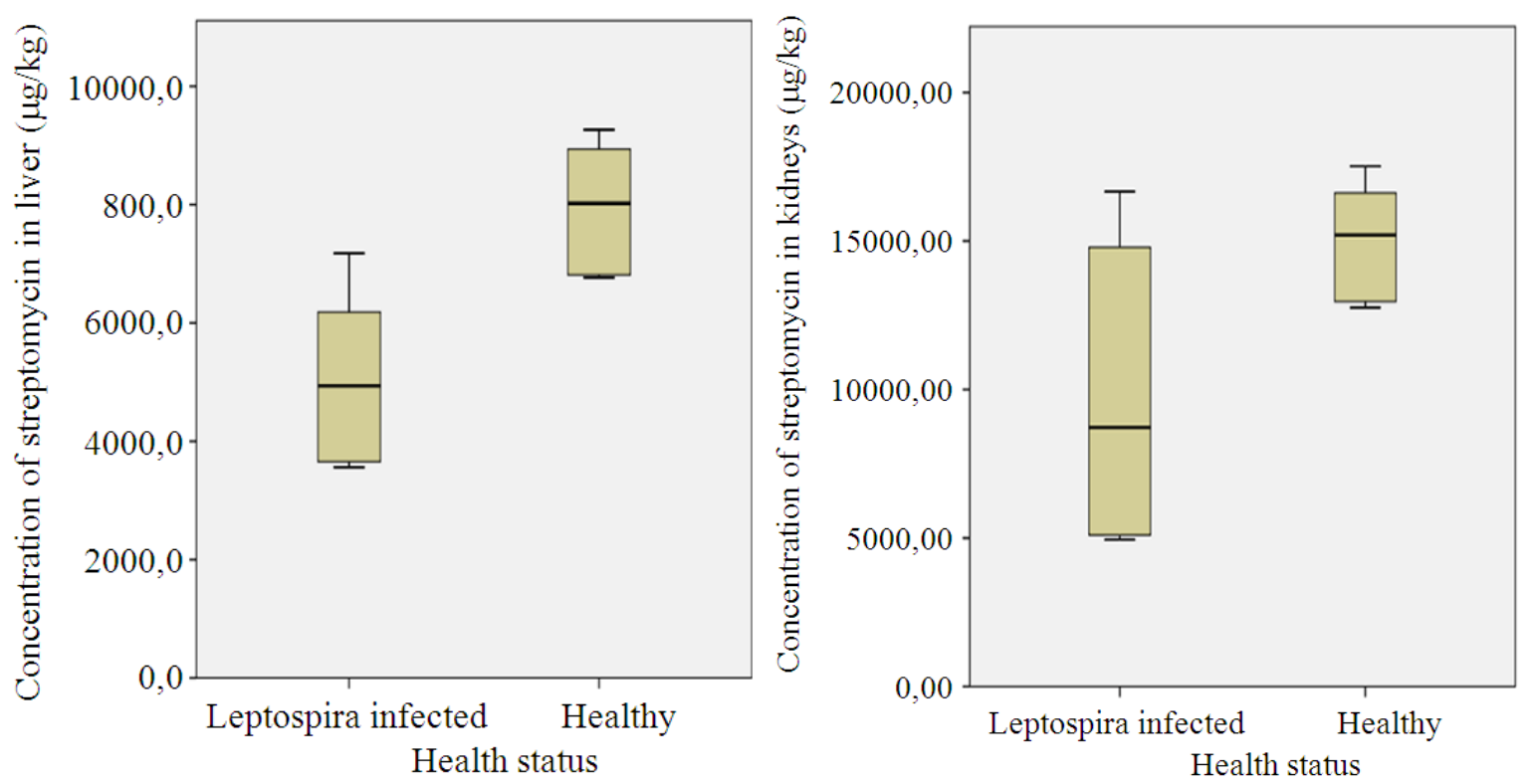

Fig. 1. Mean concentration of streptomycin \pm standard deviation in liver and kidneys of healthy pigs and pigs with leptospirae

Table 1. Biochemical profile of infected and healthy pigs

\begin{tabular}{lccc}
\hline Biochemical & $\begin{array}{l}\text { Infected mean } \\
\text { (standard deviation) }\end{array}$ & $\begin{array}{l}\text { Healty mean } \\
\text { (standard deviation) }\end{array}$ & $\begin{array}{l}\text { Reference intervals } \\
\text { (Vet Test idexx) }\end{array}$ \\
\hline BUN mg/dL & 25.17 & 21.00 & $6-30$ \\
& $(2.64)$ & $(4.19)$ & $0.5-2.1$ \\
Creatinine mg/dL & 1.50 & 1.45 & $9-43$ \\
Alanine aminotransferase U/L & $(0.32)$ & 44.17 & $92-294$ \\
Alkaline phosphatase U/L & 74.34 & $(14.48)$ & $0.1-0.3$ \\
Total Bilirubin mg/dL & $(5.39)$ & 223.33 & $(80.31)$ \\
\end{tabular}

Table 2. Concentrations of streptomycin in liver and kidneys of Leptospira spp. Infected and healthy pigs

\begin{tabular}{lllll}
\hline $\begin{array}{l}\text { Health } \\
\text { status }\end{array}$ & $\begin{array}{l}\text { No of } \\
\text { pigs }\end{array}$ & Organ & $\begin{array}{l}\text { Mean streptomycin } \\
\text { concentration }(\mu \mathrm{g} / \mathrm{Kg})\end{array}$ & $\begin{array}{l}\text { Standard } \\
\text { deviation }\end{array}$ \\
\hline Infected & 6 & Liver & $5075.2^{\mathrm{a}}$ & 1463.6 \\
Healthy & 6 & Liver & $7971.1^{\mathrm{b}}$ & 1161.8 \\
Infected & 6 & Kidneys & $9825.9^{\mathrm{c}}$ & 5072.1 \\
Healthy & 6 & Kidneys & $15045.6^{\mathrm{c}}$ & 1947.8 \\
\hline
\end{tabular}

a,b,c Figures with different superscripts differ significantly $(\mathrm{p}<0.05)$

All samples of the healthy pigs were negative. Infected pigs showed hyperbilirubinaemia and an increase in aspartate aminotransferase and alkaline phosphatase activities compared with uninfected controls. Urine specific gravity was reduced in infected pigs without elevated levels of urea and creatinine (Table 1). Mean streptomycin concentrations in liver and kidneys of infected and healthy animals are presented in Table 2 and Fig. 1. A statistically significant difference was found between the mean concentration of streptomycin in the liver of infected and healthy animals $(\mathrm{p}=0.009)$ whereas this difference was not statistically significant in kidneys $(p=0.132)$.

\section{CONCLUSION}

Although conclusions cannot be drawn about the efficacy of streptomycin against Leptospira based on PCR results since DNA can be detected even from killed pathogens the presence of leptospirae in the liver and 
kidneys of seropositive pigs is evidence of its presence after treatment. In animals the absorption of streptomycin is rapid after intramuscular administration. In cattle, peak serum levels were obtained $1 \mathrm{~h}$ after intramuscular injection of streptomycin whereas serum concentrations produced in sheep and horses paralleled those obtained in cattle (Hammond, 1953). As a result, most of parenteral dose is recovered in the urine. However, if kidney function is severely impaired, little of an intramuscular administered dose is excreted in the urine (Rollins et al., 1972; Teske et al., 1972). In the present study, reduced special gravity is indicative of renal damage and has been previously connected with leptospirotic renal lesions (Cesar et al., 2012). Although the concentrations of urea nitrogen and creatinine were not elevated in infected pigs compared to non-infected this is not sufficient to exclude kidney damage since abnormally high values appear after loss of at least 65$75 \%$ of renal functional mass.

The low drug concentration found is an indication of the effect of the disease on drug disposition and could be responsible for chronic carriage. Leptospirae cause renal tubular necrosis (Baker et al., 1989), hepatocellular damage due to the damage in the endothelium of small blood vessels (Van Den Ingh and Hartman, 1986). Liver plays a role on drug disposition primarily through its effect on protein binding and drug metabolism increasing the water solubility of a drug and facilitating its excretion in the kidney (Verbeeck, 2008). Therefore, the decreased concentrations of streptomycin observed in the present study could be at least partially attributed to the hepatic and renal damage caused by leptospirae and in turn the possible reduced efficacy of streptomycin to its reduced concentrations at the main sites of infection.

The possible effect of liver and kidney damage caused by leptospirae in drug disposition should be taken into consideration in order to define optimal dosage against the pathogen and avoid carriages.

\section{REFERENCES}

Alt, D.P. and C.A. Bolin, 1996. Preliminary evaluation of antimicrobial agents for treatment of Leptospira interrogans serovar pomona infection in hamsters and swine. Am. J. Vet. Res., 57: 59-62. PMID: 8720239

Baker, T.F., S.A. McEwen, J.F. Prescott and A.H. Meek, 1989. The prevalence of leptospirosis and its association with multifocal interstitial nephritis in swine at slaughter. Am. J. Vet. Res., 53: 290-294. PMID: 2766150
Bolin, C.A. and J.A. Cassells, 1990. Isolation of Leptospira interrogans serovar bratislava from stillborn and weak pigs in Iowa. J. Am. Vet. Assoc., 196: 1601-1604. PMID: 2189848

Bolin, C.A. and J.A. Cassells, 1992. Isolation of Leptospira interrogans serovars bratislava and hardjo from swine at slaughter. J. Vet. Diagn Inv., 4: 87-89. PMID: 1554777

Burriel, A.R., C. Dalley and M.J. Woodward, 2003. Prevalence of leptospira species among farmed and domestic animals in Greece. Vet. Rec., 153: 146148. PMID: 12934797

Cesar, K.R., E.C. Romero, A.C. De-Braganc, R.M. Blanco and P.A.E. Abreu et al., 2012. Renal involvement in leptospirosis: The effect of glycolipoprotein on renal water absorption. PLoS One, 7: e37625-e37625. DOI: 10.1371/journal.pone.0037625

Chakraborty, A., A. Takade, S.Y. Villanueva and S. Yoshida, 2011. Electron microscopic study of the effects of antimicrobial agents on the cellular architecture of Leptospira. J. Infect. Chemother., 17: 872-875. PMID: 21597899

Dobson, K.J., 1974. Letter: Eradication of leptospirosis in commercial pig herds. Austr. Vet. J., 50: 471-471. PMID: 4447531

Gravekamp, C., H.V.D. Kemp, M. Franzen, D. Carrington and G.J. Schoone et al., 1993. Detection of seven species of pathogenic leptospires by PCR using two sets of primers. J. Gen. Microbiol., 139: 1691-1700. PMID: 8409911

Hammond, P.B., 1953. Dihydrostreptomycin dose-serum level relationships in cattle. J. Am. Vet. Med. Assoc., 122: 203-206. PMID: 13044665

Levett, P.N., 2001. Leptospirosis. Clin. Microbiol. Rev., 14: 296-326.

Naigowit, P., P. Wangroongsarb, W. Petkanchanapong, O. Luepaktra and P. Warachit, 2000. A comparative evaluation of different methods for the serological diagnosis of leptospirosis. J. Trop. Med. Parasitol., 23: 59-65.

Rollins, L.D., R.H. Teske, R.J. Condon and G.G. Carter, 1972. Serum penicillin and dihydrostreptomycin concentrations in horses after intramuscular administration of selected preparations containing these antibiotics. J. Am. Vet. Med. Assoc., 161: 490495. PMID: 4115278 
Teske, R.H., L.D. Rollins and G.G. Carter, 1972. Penicillin and dihydrostreptomycin serum concentrations after administration in single and repeated doses to feeder steers. J. Am. Vet. Med. Assoc., 160: 873-878. PMID: 4110836

Van Den Ingh, T.S. and E.G. Hartman, 1986. Pathology of acute Leptospira interrogans serotype icterohaemorrhagiae infection in the Syrian hamster. Vet. Microbiol., 12: 367-376. DOI: 10.1016/0378-1135(86)90086-6
Verbeeck, R.K., 2008. Pharmacokinetics and dosage adjustment in patients with hepatic dysfunction. Eur. J. Clin. Pharmacol., 64: 1147-1161. DOI: 10.1007/s00228-008-0553-z 